\title{
Prospective implementation of a same-day discharge protocol for catheter ablation of paroxysmal atrial fibrillation
}

\author{
Anil Rajendra ${ }^{1}$ (D) $\cdot$ Tina D. Hunter ${ }^{2} \cdot$ Gustavo Morales $^{1} \cdot$ Jose Osorio $^{1}$ \\ Received: 12 August 2020 / Accepted: 8 November 2020 / Published online: 21 November 2020 \\ (C) Springer Science+Business Media, LLC, part of Springer Nature 2020
}

\begin{abstract}
Purpose Overnight stays associated with catheter ablation (CA) for paroxysmal atrial fibrillation (PAF) account for a significant proportion of treatment cost. Same-day discharge (SDD) after CA may be attractive to both patients and hospitals, especially in light of current restrictions on overnight stays due to COVID-19. This study reports on the selection criteria, protocol, and safety of SDD after CA of PAF.

Methods Patients undergoing CA for PAF were evaluated to assess the risk of groin, respiratory, cardiac, or bleeding complications. SDD eligibility criteria were stable anticoagulation with no bleeding history, systolic heart failure, respiratory conditions, or interventional procedures within 60 days, and recommended $\mathrm{BMI}<35$. Patient proximity to the hospital was also considered. Anesthesia with propofol was used, and ablations were performed with a contact force catheter. Patients rested for $6 \mathrm{~h}$ postprocedure and then ambulated over 1-2 h. Discharge followed if they were stable without evidence of complications. A nurse called all patients the following morning to elicit evidence of complications.

Results Of 44 planned SDD procedures between April 2017 and June 2018, 41 resulted in SDD after $7.2 \pm 1.0 \mathrm{~h}, 2$ patients stayed overnight for observation, and one by choice. Average age was $59 \pm 10$ years with $\mathrm{CHA}_{2} \mathrm{DS}_{2}$-VASc of $1.6 \pm 1.1$. No SDD-related complications occurred, and no return visits resulted from the follow-up calls.

Conclusion Appropriate low-risk patients identified by well-defined clinical criteria can be safely discharged the same day after CA for PAF. Evaluation in a larger population across different centers is required for generalizability of this SDD protocol.
\end{abstract}

Keywords Atrial fibrillation $\cdot$ Catheterablation $\cdot$ Same-day discharge $\cdot$ Same-day discharge protocol $\cdot$ Low-risk patients $\cdot$ Day case ablation

\section{Introduction}

Catheter ablation for atrial fibrillation (AF) has become one of the most commonly performed electrophysiologic procedures, and it accounts for a significant proportion of the overall cost for the treatment of AF [1]. In recent years, a notable decrease in complication rates for AF ablation cases have been observed [2], leading to an increasing proportion of the procedures performed in an outpatient setting, commonly with overnight monitoring for complications [3]. Ignacio et al. observed that most ablation-related complications were detected

Anil Rajendra

arajendra@alcardio.com

1 Arrhythmia Institute at Grandview, Grandview Medical Center, 3686 Grandview Parkway, Suite 720, Birmingham, AL 35243, USA

2 Real World Evidence, CTI Clinical Trial \& Consulting Services, 100 E Rivercenter Blvd, Covington, KY 41011, USA during or shortly after the ablation procedure, with only a minority of cases occurring several hours after the procedure [4]. Thus, for low-risk populations, same-day discharge (SDD) after AF catheter ablation may be an attractive strategy for both patients and hospitals, leading to both increased patient comfort and reduced hospital costs. In addition, the current COVID-19 pandemic has resulted in many hospitals implementing restrictions on overnight stays, significantly delaying elective procedures for many patients. The ability to prospectively identify AF ablation patients suitable for SDD may diminish the impact of these restrictions, allowing timely intervention for AF patients.

Limited literature exists concerning the feasibility and safety of SDD after any type of cardiac catheter ablation procedures, and all but a handful of recent publications pertain to procedures that do not involve transseptal puncture. Existing studies on SDD after AF ablation are limited to sites outside of the USA, with a single recent exception, and none report on protocols that outline selection criteria for appropriate low- 
risk patients at the time the procedure is scheduled [1, 4-7]. The objective of the current study was to report on the feasibility and safety of an SDD protocol for paroxysmal AF (PAF) ablation adopted by a high-volume site. This protocol details a set of eligibility criteria used to identify appropriate candidates from among those being scheduled to have radiofrequency catheter ablation of their PAF. The benefits of identifying patients a priori for SDD include allowing the patient to agree to the plan as well as allowing both the patient and hospital to plan accordingly.

\section{Methods}

The current study was a real-world cohort analysis, based on patient-level data collected for PAF catheter ablation procedures performed at a single high-volume site between January 2017 and June 2018. Data was collected prospectively through standardized collection forms. Beginning in April 2017, patients undergoing catheter ablation for PAF who were identified as low risk were evaluated for SDD eligibility per the site-developed protocol. SDD evaluation was performed at a pre-procedure visit, consisting of a risk assessment for groin, respiratory, cardiac, and bleeding complications. All patients were treated according to standard clinical practice and were ablated by one of three operators experienced in radiofrequency (RF) ablation.

Baseline patient characteristics, procedure-related complications, measures of procedural efficiency, and 3-month clinical effectiveness outcomes were collected for each ablation. Approval from the local institutional review board was obtained.

\subsection{Identification of study cohorts}

Patients pre-identified for the SDD cohort were required to meet the following eligibility criteria per the SDD protocol (Table 1): (1) stable anticoagulation (stable therapeutic INR within 1 month before procedure if on warfarin or patient reported compliance with direct oral anticoagulant), (2)

Table 1 SDD protocol eligibility criteria

SDD eligibility criteria

1. Stable anticoagulation

2. Absence of bleeding history

3. No systolic heart failure

4. No history of pulmonary disease

5. No interventional procedures within 60 days of ablation

6. $\mathrm{BMI}<35$ (recommended)

7. Acceptable $\mathrm{CHA}_{2} \mathrm{DS}_{2}$-VASc stroke risk (typically $\leq 3$ ) absence of bleeding history, (3) no systolic heart failure, (4) no history of pulmonary disease, (5) no interventional procedures within 60 days of ablation, (6) $\mathrm{BMI}<35$ (recommended), and (7) acceptable $\mathrm{CHA}_{2} \mathrm{DS}_{2}-\mathrm{VASc}$ stroke risk (typically $\leq 3$ ). The patients' proximity to the hospital and willingness to stay at a hotel nearby, if needed, were also considered. Eligible patients were then scheduled for ablations prior to 1:00 PM.

In addition to the eligibility criteria laid out in the protocol, patients included in the SDD cohort for this analysis also met the following inclusion criteria: (1) $\geq 21$ years of age, (2) ablated for PAF between April 2017 (when the SDD protocol was adopted) and June 2018, and (3) ablated with a porous tip contact force $(\mathrm{CF})$ catheter. Similar adults who were ablated for PAF in 2017, but who were held overnight, were also included in the study and analyzed as a comparator cohort (non-SDD).

\subsection{Ablation procedures}

All patients underwent ablation on uninterrupted oral anticoagulation, including taking it on the morning of the procedure, and all ablations were performed with a THERMOCOOL SMARTTOUCH® SF Catheter (Biosense Webster, Inc., Irvine, CA). The porous tip catheter was used to minimize fluid delivery during the procedure. General anesthesia with propofol was administered. The use of ultrasound to guide venous access was at the operator's preference. Pulmonary veins were isolated by wide area circumferential ablation using catheter stability software (CARTO VISITAG ${ }^{\mathrm{TM}}$ Module, Biosense Webster, Inc.) and a previously described standardized workflow. [8] Ablation of the cavotricuspid isthmus was left to the discretion of the operator.

Following the procedure, patients were on bed rest for $6 \mathrm{~h}$ and then ambulated intermittently for $1-2 \mathrm{~h}$. For patients in the SDD cohort, discharge followed if they were stable with no evidence of complications after ambulation. The specific discharge criteria required in the SDD protocol (Table 2), and recorded for every SDD patient, were as follows: (1) the procedure occurred without complications, (2) post-procedure

Table 2 SDD protocol discharge criteria

Discharge Criteria

1. Procedure occurred without complications

2. Post-procedure confirmation by operator to proceed with SDD

3. Purse string suture removed

4. Stable hemodynamics

5. No evidence of groin or respiratory complications

6. Able to tolerate liquids/food

7. Able to ambulate 
confirmation was obtained from the operator to proceed with SDD, (3) the purse string suture removed, (4) the patient had stable hemodynamics, (5) there was no evidence of groin or respiratory complications, (6) the patient was able to tolerate liquids/food, and (7) the patient was able to ambulate.

\subsection{Patient follow-up}

All patients were monitored for complications during the procedure and again pre-discharge. Visual inspection was performed of bilateral groins to ensure adequate hemostasis and no signs of vascular access complications. All patients were discharged with colchicine for 2 weeks to prevent pericarditis. The morning after the procedure, patients in the SDD cohort were telephoned by a dedicated nurse and asked a series of standard questions designed to elicit evidence of any complications. On the seventh day after the procedure, all patients (SDD and non-SDD) were telephoned by a nurse to elicit evidence of complications. Patients from both cohorts were scheduled for a 3-month follow-up visit in order to assess the safety and effectiveness of their respective procedures. During this visit, a transthoracic echocardiogram (TTE) was performed to screen for pericardial effusion.

\subsection{Study endpoints}

The primary outcome of interest for the current study was validation of the SDD protocol adopted by the site through verification that patients chosen for SDD were indeed likely to be able to go home on the day of their ablation, as planned, and that patients discharged on the same day were not compromised with respect to safety or effectiveness when compared with the non-SDD cohort. Of secondary interest were procedural efficiency outcomes, which were not expected to be affected by SDD status.

\subsection{Cohort matching}

All patients who were identified as SDD candidates, who then accepted the offer of SDD and were subsequently discharged as planned, comprised the SDD cohort. All patients who were ablated for PAF with an STSF catheter in 2017, and who were not identified for the SDD cohort, were eligible for the nonSDD cohort. From the batch of eligible non-SDD patients, those with clinical characteristics outside of the range allowed by the SDD protocol were excluded from consideration prior to matching. These exclusions included patients who were (1) 75 years of age or older, (2) had a $\mathrm{CHA}_{2} \mathrm{DS}_{2}$-VASc score $>4$, or (3) had a BMI $\geq 40$. Patients from the SDD cohort were then matched 1:1 with similar patients in the non-SDD cohort using the SAS gmatch macro, developed by Kosanke and Bergstralh [9]. The final non-SDD cohort was matched to the SDD cohort with respect to the following criteria: (1) operator, (2) sex, (3) diabetes, (4) $\mathrm{CHA}_{2} \mathrm{DS}_{2}$-VASc score within 1 point, and (5) availability of $10-12$ week visit data.

\section{Results}

\subsection{Patient characteristics}

A total of 52 ablations met all eligibility and inclusion criteria for SDD, with 7 patients declining the offer of SDD at the time of scheduling. One additional patient was then excluded due to missing SDD protocol data as a result of late identification. Of the remaining 44 procedures, 41 (93.2\%) resulted in discharges on the same day, thus comprising the SDD cohort (see Fig. 1). Two patients had two ablations each that were both included in the SDD cohort.

The 41 procedures in the resulting SDD cohort were successfully matched to 41 non-SDD procedures performed in patients with similar baseline characteristics to create the full analysis population. The final SDD and non-SDD cohorts were similar with respect to baseline characteristics (see Table 3).

\subsection{Outcomes}

Three patients who were eligible for SDD post-procedure were not discharged as planned. One decided to stay overnight for comfort, one was held for observation of a fever, and the third for observation of groin bleeding. All three patients were discharged the following morning. The remaining SDD patients were discharged after a mean recovery time of $7.2 \pm$ $1.0 \mathrm{~h}$.

There were no major complications observed in patients identified for SDD. Two SDD patients had minor right groin hematomas and were discharged on the day of the procedure, as planned. All patients in the SDD group were contacted by a nurse the day after the procedure to ask questions about potential complications and assess for need to return to the hospital for evaluation. None of the patients had any complications or need to return to hospital or clinic for evaluation.

Two patients in the non-SDD cohort also had recorded complications. While they were significant, both were related to underlying conditions rather than the procedure itself. One of the non-SDD patients had blood in their stool and rectal bleeding that began approximately 4 days post-procedure and resulted in transfusion of 2 units of packed red blood cells at 79 days post-ablation. The second patient had an AV block requiring a temporary pacemaker placement on the day of the procedure.

Mean procedure times were $59 \pm 15 \mathrm{~min}$ for the SDD cohort and $67 \pm 22 \mathrm{~min}$ for the non-SDD cohort (Table 4). The majority of patients were ablated without the use of fluoroscopy (90.2\% in SDD cohort, $87.7 \%$ in non-SDD cohort), 


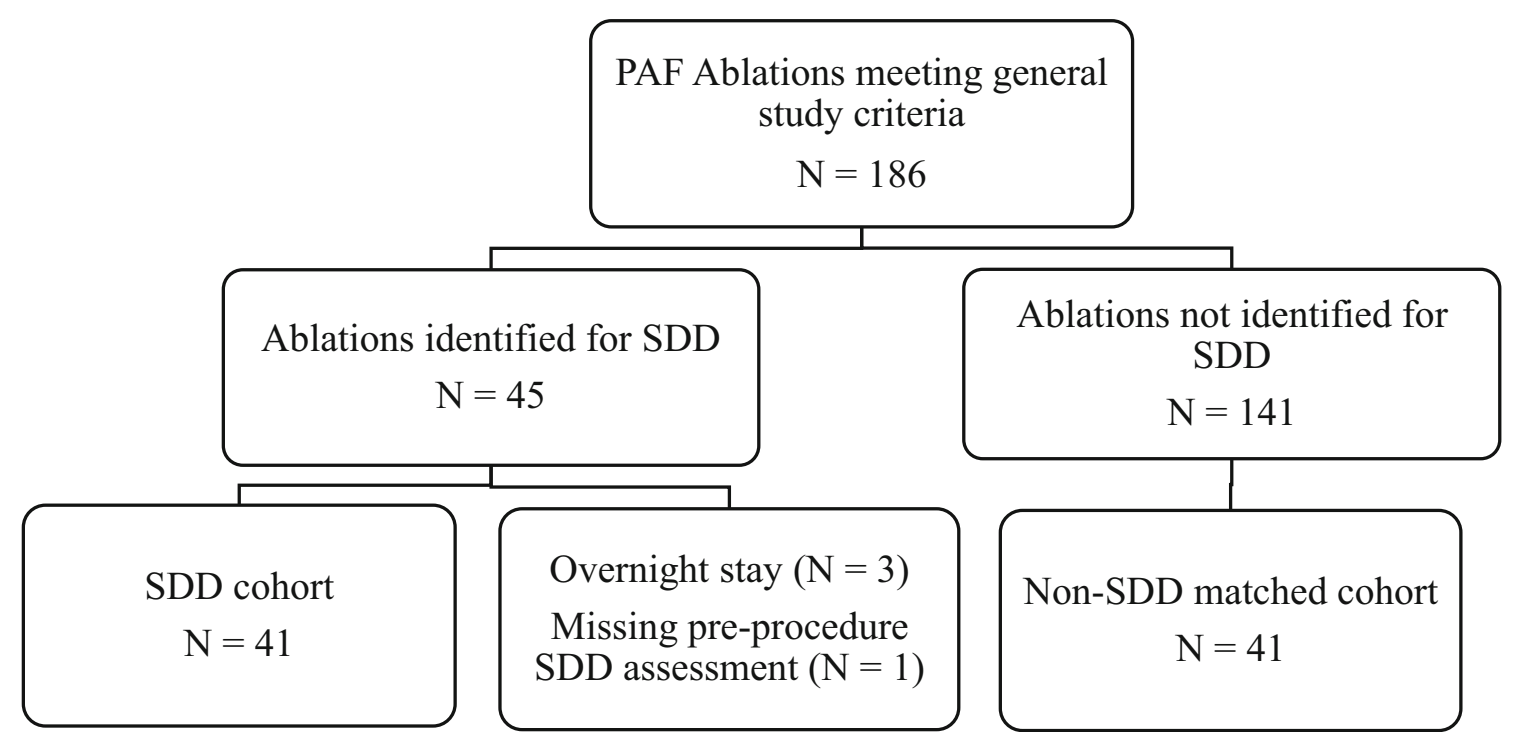

Fig. 1 Analysis population

resulting in mean fluoroscopy usage of just $0.01 \pm 0.04 \mathrm{~min}$ and $0.02 \pm 0.07 \mathrm{~min}$ in the SDD and non-SDD cohorts,

Table 3 Baseline patient characteristics

\begin{tabular}{lll}
\hline Patient characteristic & SDD $(N=41)$ & Non-SDD $(N=41)$ \\
\hline Gender = male & $25(61.0 \%)$ & $25(61.0 \%)$ \\
Age, mean \pm SD & $58.5 \pm 9.9$ & $59.9 \pm 12.8$ \\
Age category & & \\
$<65$ years & $26(63.4 \%)$ & $25(61.0 \%)$ \\
$65-74$ years & $15(36.6 \%)$ & $16(39.0 \%)$ \\
$\geq 75$ years & $0(0.0 \%)$ & $0(0.0 \%)$ \\
Body mass index (BMI) & $30.1 \pm 5.2$ & $29.7 \pm 4.6$ \\
Congestive heart failure & $2(4.9 \%)$ & $0(0.0 \%)$ \\
Hypertension & $23(56.1 \%)$ & $23(56.1 \%)$ \\
Diabetes & $1(2.4 \%)$ & $1(2.4 \%)$ \\
Stroke/TIA/TE & $2(4.9 \%)$ & $0(0.0 \%)$ \\
Vascular disease & $3(7.3 \%)$ & $8(19.5 \%)$ \\
Renal disease & $0(0.0 \%)$ & $0(0.0 \%)$ \\
Liver disease & $0(0.0 \%)$ & $0(0.0 \%)$ \\
Known prior ablation & $3(7.3 \%)$ & $2(4.9 \%)$ \\
CHA 2 DS ${ }_{2}$-VASc score & & \\
0 & $8(19.5 \%)$ & $8(19.5 \%)$ \\
1 & $11(26.8 \%)$ & $13(31.7 \%)$ \\
2 & $14(34.1 \%)$ & $10(24.4 \%)$ \\
3 & $7(17.1 \%)$ & $9(22.0 \%)$ \\
4 & $1(2.4 \%)$ & $1(2.4 \%)$ \\
HAS-BLED score & $(N=27)$ & $(N=19)$ \\
0 & $10(37.0 \%)$ & $6(31.6 \%)$ \\
1 & $12(44.4 \%)$ & $8(42.1 \%)$ \\
2 & $4(14.8 \%)$ & $5(26.3 \%)$ \\
3 & $1(3.7 \%)$ & $0(0.0 \%)$ \\
\hline & & \\
\hline & &
\end{tabular}

BMI was only available for 18 patients in the non-SDD cohort respectively. At the 3-month follow-up visit, there were two early recurrences of AF (4.9\%) observed in each cohort.

\section{Discussion}

The primary finding of the current study is that the SDD protocol criteria successfully identified PAF patients who could be safely discharged on the same day as their ablation procedure. This is encouraging, as SDD may be economically beneficial to hospitals as well as beneficial to patients who would be more comfortable in their own beds overnight. To our knowledge, this is the first study to report on specific protocol criteria for pre-identifying an appropriate SDD population as well as for discharging the chosen patients after AF ablation.

Despite the absence of details on specific selection and discharge criteria, four publications have reported on the experiences of sites beginning to offer SDD after AF ablation in the UK, Buenos Aires, Canada, and the USA. Several additional publications have reported on findings from studies of SDD in catheter ablation populations that excluded AF ablation.

Bartolleti et al. recently reported on SDD for AF ablation patients at a tertiary center in the UK [5]. All patients scheduled for AF ablation in the morning, with procedures ending before 2 PM, were considered for SDD. Procedures were performed with either a contact force catheter or a cryoballoon and included ablations performed under either general anesthesia or conscious sedation. The option for SDD was not presented to patients until they were reviewed by an electrophysiologist after the procedure. Of the morning cases considered for SDD, $20.8 \%$ were discharged on the same day. Among the day case cohort, 1 patient $(0.7 \%)$ experienced a procedural phrenic nerve palsy with confirmed resolution prior to discharge, $3.5 \%$ experienced minor complications, and $2.1 \%$ required rehospitalization after 
Table 4 Procedural efficiency and early effectiveness

\begin{tabular}{lll}
\hline Procedural efficiency measure & SDD $(N=41)$ & Non-SDD $(N=41)$ \\
\hline Total procedure time (min) & $59 \pm 15$ & $67 \pm 22$ \\
Radiofrequency time (min) & $27 \pm 6$ & $30 \pm 11$ \\
PV ablation time (min) & $20 \pm 5$ & $22 \pm 9$ \\
Time to isolate LEFT PVs (min) & $4.2 \pm 2.1$ & $4.4 \pm 2.4$ \\
Time to isolate RIGHT PVs (min) & $7.0 \pm 3.0$ & $7.5 \pm 3.9$ \\
Total fluoroscopy time (min) & $0.01 \pm 0.04$ & $0.02 \pm 0.07$ \\
No fluoroscopy used & $37(90.2 \%)$ & $36(87.7 \%)$ \\
Radiation dose (mGy) & $0.6 \pm 0.9$ & $0.9 \pm 1.2$ \\
IV fluids (mL) & $281 \pm 78$ & $300 \pm 103$ \\
Catheter fluids (mL) & $508 \pm 144$ & $538 \pm 210$ \\
Total fluid delivered (mL) & $793 \pm 184$ & $879 \pm 228$ \\
Early atrial arrhythmia recurrence & & $2(4.9 \%)$ \\
Recurrence recorded at 3-month visit & $2(4.9 \%)$ & \\
\hline
\end{tabular}

discharge for symptoms treatable with conservative management. While the authors experienced success with SDD after AF ablation, and ultimately the criteria used to select eligible patients was likely similar to our study, none of the criteria were applied a priori. In fact, the authors discuss as a limitation that operators could decide whether to offer SDD to patients having morning ablations without using pre-specified criteria to guide the decision. If a standard set of criteria had been applied earlier, at the time of scheduling, the low-risk patients could have been scheduled as morning cases, potentially doubling the number of resulting day cases.

Opel et al. also reported on the feasibility of SDD after AF ablation at a UK center. [1] In this case, staff at a small local noncardiac center were trained to support high-throughput AF ablation procedures performed as day cases by operators from a regional cardiac center. Results from the local center were compared with those from matched patients ablated at the regional cardiac center, where patients were routinely held overnight. Patients recruited to the new program included patients having de novo ablation for PAF or for persistent AF of $<1$-year duration, with exclusions for patients requiring general anesthesia or RF ablation. Of the 276 patients ablated in the local center, $5.4 \%$ experienced complications and $1.4 \%$ were not discharged on the same day. Safety and effectiveness were similar at the local and regional centers. The more limited facilities at the center performing the day cases necessitated a plan for transfer of patients with serious complications that would not be necessary in a larger hospital. The results from this study are complimentary to the current study in that they included only cryoballoon ablations, while the current study included only RF ablations.

The third study to report on SDD after AF ablation did so for a cohort of consecutive patients receiving pulmonary vein isolation (PVI) at a site in Buenos Aires that included both RF and cryoballoon ablations [4]. There were 205 consecutive procedures included, with $10(4.9 \%)$ patients held overnight for complications. Of the remaining 195 patients, $29.7 \%$ were discharged on the same day as their procedure. No information was presented to discern how those patients were chosen for SDD. None of the 10 complications that occurred during the observation period first presented after the 6th hour, and most were detected during the procedure. Significantly fewer visits to the emergency room were seen at 30 days in the SDD group versus in the overnight group (15.5\% vs. $30.7 \%)$.

Deyell et al. [7] reported that $79.2 \%$ of 3054 patients who met inclusion criteria and were ablated at two Canadian centers between 2010 and 2014 were discharged on the same day as their $\mathrm{AF}$ ablation. In addition, they reported that the primary reasons for later discharge were access site problems $(22.8 \%)$, late procedure finish $(23.9 \%)$, intraprocedural complications (10.1\%), and anesthesia-related problems $(8.8 \%)$. A multivariable analysis confirmed that patient age, female sex, intraprocedural complication, and a procedure ending after 1:00 PM were associated with increased likelihood of admission. Being able to identify the patients at risk of complications ahead of time and scheduling low-risk patients for earlier procedures could provide further advantages for both patients and hospitals due to increased predictability, as evidenced by the $93.2 \%$ discharge rate in the current study.

Finally, a US site recently reported on a chart review of 426 consecutive patients with elective outpatient ablations for AF or left atrial flutter after adoption of SDD (56.7\% PAF, March 2010 to December 2015) [6]. Patients were monitored for at least $6 \mathrm{~h}$ post-ablation, were reassessed prior to discharge, and were contacted by phone the next day. Of the 426 potential SDD patients, $50(12 \%)$ were not discharged on the same day, with $18(4.2 \%)$ due to patient preference, $17(4.0 \%)$ due to ablation related complications, and 15 (3.5\%) due to non-ablation-related medical care. Major complications occurred in $1.4 \%(n=6)$, including 3 pericardial effusions requiring drainage, 2 transient ischemic attacks, and 1 blood transfusion. None of the major complications 
occurred within $24 \mathrm{~h}$ of discharge. The percentage of patients discharged on the same day is likely inflated and the complication rate lowered due to the pre-exclusion of charts from patients who were admitted as inpatients. Even so, it does support the potential for safely discharging low-risk patients on the day of ablation.

Prior to the very recent studies described above, several publications reported on findings of SDD after cardiac catheter ablations that excluded $\mathrm{AF}$ ablation or any procedures requiring transseptal puncture [10-12]. Several authors noted that despite the increase in cost to maintain patients overnight, there was no apparent increase in safety [10-12]. Theodoreson et al. reported no complications that would have been detected by remaining in the hospital overnight [12].

As SDD is studied at more institutions, patient preference should remain a consideration as a discharge criterion in the development of SDD protocols. Between the initial scheduling of the procedure and the post-ablation observation period, numerous variables may affect a patient's level of comfort with early discharge or their safety outside of a hospital setting. In the current study, 7 of $52(13.5 \%)$ patients initially identified as eligible for SDD declined the offer prior to their procedure for reasons of personal preference and/or comfort and one patient decided post-procedure that they preferred to stay overnight. In addition to the proximity of their residence to the hospital, another consideration for patients may be the presence or absence of a partner in their home who is willing and able to provide for their immediate needs.

During the current COVID-19 pandemic, several publications are providing guidance for clinicians and institutions to rebuild safe electrophysiological care [13-15]. A joint guidance from the Heart Rhythm Society, the American Heart Association, and a committee within the American College of Cardiology recommends consideration of same-day discharge, as clinically appropriate, to minimize patient exposure [13]. The current study may provide a helpful guide by establishing well-defined criteria for preemptive identification of low-risk patients that can be safely discharged the same day, thus reducing their potential for exposure to COVID-19.

\subsection{Limitations}

As with any observational study, there were limitations present in the current study that are worth addressing. First, the inclusion criteria used for the SDD cohort could not be fully replicated in the non-SDD cohort due to additional risk detail that was collected only for SDD candidates. However, we believe that the observed results would be considered strong evidence of feasibility, even in the absence of a control cohort for reference. Furthermore, the current study reports on a single set of criteria for selecting SDD-eligible patients, with a single set of criteria for patient-discharge. Consequently, the reported results must be viewed only as evidence of feasibility, with respect to the specific SDD protocol, rather than evidence of having developed an optimal strategy. It is also plausible that the results of the current study were due, in part, to the experience of the operators and thus may not be generalizable to less-experienced sites. A larger study with diverse sites will be needed to determine whether this SDD protocol is more widely applicable and to accurately estimate the proportion of $\mathrm{AF}$ patients who would be eligible for this protocol.

Further research will be needed to validate the findings of this study and to further explore the optimal criteria for selecting SDD patients. Reproducibility of these results, using the outlined SDD protocol in other institutions, will also need to be tested. Future studies should aim to minimize procedure costs and maximize patient satisfaction, without compromising safety.

\section{Conclusions}

Through the adoption of well-defined criteria, it is feasible for low-risk patients to be identified and safely discharged the same day after an RF catheter ablation procedure for PAF. Further investigation is required to determine whether SDD is feasible for patient populations with different risk profiles or at sites with differing levels of experience in AF ablation.

Authors' contributions A Rajendra, G Morales, and J Osorio contributed to data acquisition, TD Hunter was responsible for the analysis and drafting of the manuscript, and all authors contributed to the concept, study design and interpretation of the data. All authors contributed to critical revision of the manuscript and have approved the final version for submission.

Funding This study was funded by Biosense Webster, Inc.

\section{Compliance with ethical standards}

Disclosures A Rajendra is a paid consultant for Biosense Webster, Inc., Abbott, Medtronic, Boston Scientific, and Philips. G Morales is a paid consultant for Biosense Webster, Inc., Abbott, Medtronic, and Boehringer Ingelheim. J Osorio is a paid consultant for Biosense Webster, Inc., and Boston Scientific. Neither honoraria nor payments were made for authorship. TD Hunter is an employee of CTI Clinical Trial and Consulting Services, which is a consultant to Biosense Webster, Inc.

\section{References}

1. Opel A, Mansell J, Butler A, Schwartz R, Fannon M, Finlay M, et al. Comparison of a high throughput day case atrial fibrillation ablation service in a local hospital with standard regional tertiary cardiac centre care. Europace. 2019;21(3):440-4. https://doi.org/ 10.1093/europace/euy153.

2. Muthalaly RG, John RM, Schaeffer B, Tanigawa S, Nakamura T, Kapur S, et al. Temporal trends in safety and complication rates of 
catheter ablation for atrial fibrillation. J Cardiovasc Electrophysiol. 2018;29(6):854-60. https://doi.org/10.1111/jce.13484.

3. Haegeli LM, Duru F, Lockwood EE, Luscher TF, Sterns LD, Novak PG, et al. Feasibility and safety of outpatient radiofrequency catheter ablation procedures for atrial fibrillation. Postgrad Med J. 2010;86(1017):395-8. https://doi.org/10.1136/pgmj.2009.092510.

4. Ignacio DM, Jarma DJJ, Nicolas V, Gustavo D, Leandro T, Milagros C, et al. Current safety of pulmonary vein isolation in paroxysmal atrial fibrillation: first experience of same day discharge. J Atr Fibrillation. 2018;11(4):2077. https://doi.org/10. 4022/jafib.2077.

5. Bartoletti S, Mann M, Gupta A, Khan AM, Sahni A, El-Kadri M, et al. Same-day discharge in selected patients undergoing atrial fibrillation ablation. Pacing Clin Electrophysiol. 2019;42(11): 1448-55. https://doi.org/10.1111/pace.13807.

6. Akula DN, Mariam W, Luthra P, Edward F, Katz DJ, Levi SA, et al. Safety of same day discharge after atrial fibrillation ablation. J Atr Fibrillation. 2020;12(5):2150. https://doi.org/10.4022/jafib. 2150.

7. Deyell MW, Leather RA, Macle L, Forman J, Khairy P, Zhang R, et al. Efficacy and safety of same-day discharge for atrial fibrillation ablation. JACC Clin Electrophysiol. 2020;6(6):609-19. https://doi. org/10.1016/j.jacep.2020.02.009.

8. Osorio J, Hunter TD, Bubien RS, Rajendra A, Arciniegas J, Morales G. Gains in paroxysmal atrial fibrillation ablation using a standardized workflow to optimize contact force technologies. J Atr Fibrillation. 2018;11(4):2097. https://doi.org/10.4022/jafib.2097.

9. Kosanke J, Bergstralh E. GMATCH SAS Macro. Mayo Clinic College of Medicine. 2007. http://bioinformaticstools.mayo.edu/ research/gmatch/. Accessed 7 Feb 2019.

10. Golia P, Bandini A, Galvani M. Same-day discharge after catheter ablation for routine arrhythmias: an initial experience. Minerva Cardioangiol. 2012;60(3):267-73.
11. Marijon E, Albenque JP, Boveda S, Jacob S, Schmutz M, Bortone A, et al. Feasibility and safety of same-day home discharge after radiofrequency catheter ablation. Am J Cardiol. 2009;104(2):2548. https://doi.org/10.1016/j.amjcard.2009.03.024.

12. Theodoreson MD, Chohan BC, McAloon CJ, Sandhu A, Lancaster CJ, Yusuf S, et al. Same-day cardiac catheter ablation is safe and cost-effective: experience from a UK tertiary center. Heart Rhythm. 2015;12(8):1756-61. https://doi.org/10.1016/j.hrthm.2015.05.006.

13. Lakkireddy DR, Chung MK, Deering TF, Gopinathannair R, Albert CM, Epstein LM, et al. Guidance for rebooting electrophysiology through the COVID-19 pandemic from the Heart Rhythm Society and the American Heart Association Electrocardiography and Arrhythmias Committee of the Council on Clinical Cardiology: endorsed by the American College of Cardiology. Heart Rhythm. 2020;17:e242-54. https://doi.org/10.1016/j.hrthm.2020.06.012.

14. Rubin GA, Wan EY, Saluja D, Thomas G, Slotwiner DJ, Goldbarg $\mathrm{S}$, et al. Restructuring electrophysiology during the COVID-19 pandemic: a practical guide from a New York City Hospital Network. Crit Pathw Cardiol. 2020;19(3):105-11. https://doi.org/ 10.1097/HPC.0000000000000225.

15. Saenz LC, Miranda A, Speranza R, Texeira RA, Rojel U, Enriquez A, et al. Recommendations for the organization of electrophysiology and cardiac pacing services during the COVID-19 pandemic : Latin American Heart Rhythm Society (LAHRS) in collaboration with: Colombian College Of Electrophysiology, Argentinian Society of Cardiac Electrophysiology (SADEC), Brazilian Society Of Cardiac Arrhythmias (SOBRAC), Mexican Society Of Cardiac Electrophysiology (SOMEEC). J Interv Card Electrophysiol. 2020;59:307-13. https://doi.org/10.1007/s10840-020-00747-5.

Publisher's note Springer Nature remains neutral with regard to jurisdictional claims in published maps and institutional affiliations. 